\title{
Case Report \\ The Treatment of Clozapine-Withdrawal Delirium with Electroconvulsive Therapy
}

\author{
Anish Modak and Arne Åhlin \\ Adult Mental Health Unit, The Canberra Hospital, ACT Health, GPO Box 825, Canberra, ACT 2601, Australia \\ Correspondence should be addressed to Anish Modak; anishmodak@gmail.com
}

Received 7 September 2017; Accepted 16 October 2017; Published 2 November 2017

Academic Editor: Toshiya Inada

Copyright (c) 2017 Anish Modak and Arne Åhlin. This is an open access article distributed under the Creative Commons Attribution License, which permits unrestricted use, distribution, and reproduction in any medium, provided the original work is properly cited.

\begin{abstract}
Clozapine, a commonly used atypical antipsychotic, can precipitate a severe withdrawal syndrome. In this report, we describe a case of delirium with catatonic features emerging after the immediate cessation of clozapine subsequent to concerns of developing neuroleptic malignant syndrome. After multiple treatments were found to be inefficacious, electroconvulsive therapy (ECT) was initiated, resulting in significant improvement. A literature search revealed six previous cases of clozapine-withdrawal syndromes of varied symptomatology treated with ECT. To our knowledge, the present case represents the first reported clozapine-withdrawal delirium treated successfully with ECT.
\end{abstract}

\section{Introduction}

We present a case of delirium with catatonic features following the abrupt cessation of clozapine treatment, which responded to electroconvulsive therapy (ECT). The complexity and diagnostic difficulty of this case highlight the overlap of symptoms between various syndromes, including delirium, catatonia, and neuroleptic malignant syndrome (NMS), all of which can be associated with clozapine withdrawal. Despite evidence from the literature that delirium following clozapine withdrawal can occur, there have been no published cases of clozapine-withdrawal delirium treated with ECT.

\section{Case Presentation}

Mr. J is a 30-year-old Australian man with schizoaffective disorder, who presented with delirium in the context of abrupt cessation of clozapine due to the development of suspected NMS.

Mr. J initially presented to hospital with an exacerbation of schizoaffective disorder, characterized by formal thought disorder and persecutory delusions. Additionally, Mr. J had mixed mood symptoms; with elements of both mania and depression associated with mood-congruent delusions of grandiosity and guilt, respectively. There had been a deterioration in the community over a period of months, with increasingly disorganised and erratic behavior, poor insight, and a decline in functioning. Mr. J was admitted to an acute psychiatric unit under an involuntary treatment order. $\mathrm{He}$ was initially treated with $1.5 \mathrm{~g}$ of sodium valproate in divided doses, in combination with $6 \mathrm{mg}$ of risperidone daily, over 5 weeks. Subsequently, he was trialed on a combination of sodium valproate, at the same dose, and $20 \mathrm{mg}$ of olanzapine daily, over 5 weeks. After failing to respond to these medications, Mr. J was commenced on clozapine in week 10 of his admission.

This occurs on the background of one previous admission with psychosis at age 24 , with similar symptoms of thought disorder and referential delusions. Mr. J's first episode of mental illness was prolonged, with slow response to risperidone treatment, and complicated by an episode of depression. After a year of recovery requiring residential care in a stepdown facility, Mr. J had a period of reasonable interepisodic function, working in a call-centre and living independently, on a treatment regimen of low dose oral risperidone and venlafaxine. He is supported by his family, does not use tobacco, alcohol, or other drugs, and has an unremarkable medical history otherwise. There is a family history of bipolar disorder in his mother. 
On week 6 of clozapine initiation, after reaching a dose of $300 \mathrm{mg}$ daily and over a few days, Mr. J developed persistent tachycardia (heart rate up to 130), hypertension (systolic blood pressure up to $168 \mathrm{mmHg}$ ), low grade fevers $\left(37.8^{\circ} \mathrm{C}\right)$, and rigidity, associated with neutrophilia (up to $9.44 * 10^{9} / \mathrm{L}$ ) and a raised creatine kinase (CK) (up to $5870 \mathrm{U} / \mathrm{L}$ ). At this time, he was prescribed no other medication. Mr. J was treated for a presumptuous diagnosis of NMS with abrupt cessation of clozapine and intravenous fluids on a general medical ward, prior to transfer back to the acute psychiatric unit following resolution of his fevers and autonomic symptoms.

Over the following days, Mr. J deteriorated rapidly in his mental state, with floridly disorganised thoughts (word salad), both increased and decreased psychomotor activity (singing, hugging staff, and statuesque posture), severe insomnia (no sleep for 3 days), visual hallucinations, fluctuating disorientation and inattention, and urinary incontinence. Physical examination revealed negativism, intermittent rigidity, and generalised hyperreflexia, but no clonus, autonomic dysfunction, diaphoresis, or fevers. He required 1:1 nursing care and prompting for food and fluid intake. This profoundly delirious, catatonic, and psychotic state failed to respond to oral lorazepam (up-titrated to $2 \mathrm{mg}$ four times a day) or valproate (1.5 g daily in divided doses). Chloral hydrate was trialed for insomnia, with insufficient effect. Additionally, Mr. J received a brief trial of benztropine to treat possible cholinergic rebound symptoms, again, with no apparent benefit. Antipsychotic treatment was withheld due to the concern of NMS. CK levels continued to fluctuate, though slowly resolved over two weeks. A lumbar puncture was performed under sedation, with normal protein and cell count in the cerebrospinal fluid, and no evidence of antineuronal antibodies to suggest autoimmune encephalitis. Other serum investigations, including an ammonia level to exclude valproate-induced hyperammonaemic encephalopathy, were unremarkable. As Mr. J had had a normal magnetic resonance imaging- (MRI-) brain earlier in his admission, cerebral imaging was not repeated.

Electroconvulsive therapy (ECT) was commenced on day 13 after clozapine cessation after obtaining an ECT treatment order. Mr. J's first two ECT treatments were delivered bifrontally in order to minimize cognitive side-effects; subsequently, he received a course of 8 further bitemporal ECT treatments, at a pulse width of $1 \mathrm{~ms}$, with dramatic improvement in his mental state observed from the 4 th treatment. His self-care and function improved, and he was able to be managed in a low-dependency environment without $1: 1$ nursing. During this time, lorazepam and valproate treatment were ceased due to their anticonvulsant effects. Mr. J was transferred to a psychiatric rehabilitation facility 4 weeks after his first ECT treatment, with some residual psychotic symptoms, having been initiated on a regimen of lithium and quetiapine.

\section{Discussion}

In the literature, there have only been a few case reports of clozapine-withdrawal syndromes treated with ECT. It is well established that abrupt cessation of clozapine can precipitate a rebound psychosis or mania $[1,2]$. However, rarer syndromes have also been associated with clozapine withdrawal, such as delirium [3], catatonia [4], serotonin syndrome [5], NMS [6], and movement disorders such as dystonias and dyskinesias [7]. The diverse presentation of clozapine-withdrawal states is likely secondary to its broad pharmacological profile, with antagonistic effects in serotonergic, cholinergic, dopaminergic, and histaminergic receptors leading to rebound hyperactivity in these systems, along with rebound dysregulation of gamma-aminobutyric-acid(GABA-) ergic pathways as a result of clozapine's activity on GABA receptors $[4,8,9]$. Treatment of clozapine-withdrawal syndromes should be targeted at particular neurotransmitter systems depending on the symptoms and signs observed; for example, benztropine and cyproheptadine have been suggested as treatments for cholinergic and serotonergic rebound symptoms, respectively $[5,9]$. Using an antipsychotic with a high anticholinergic potency, such as chlorpromazine, may reduce the risk of cholinergic delirium when clozapine needs to be stopped abruptly $[3,9]$.

A recent literature review by Bilbily and colleagues revealed 7 cases of clozapine-withdrawal catatonia reported previously, 2 of which were treated successfully with ECT [4]. For the present review, a literature search was undertaken in April 2017, using PubMed, Medline, and PsycInfo databases using the search terms "Clozapine" AND "Electroconvulsive" AND "Rebound" OR "Withdrawal" OR "Cessation." An additional 4 further case reports were identified in which clozapine-withdrawal syndromes were treated with ECT. Of the total 6 cases identified, 4 were described as cases of catatonia, 1 was described as a case of mania, and 1 was described as a case of rebound psychosis and NMS, in both males and females aged between 26 and 58 (Table 1). There were no cases of clozapine rebound delirium treated with ECT. One case of clozapine-withdrawal catatonia had no response to ECT; otherwise, the other case reports described partial or full response to treatment. However, it is possible that negative results from trials of ECT in clozapine-withdrawal have not been reported in the literature. Finally, the particular modalities of ECT used were not mentioned in any of the articles (e.g., bitemporal or unilateral).

In the present case, it was clear that a dramatic and profound alteration in mental status was temporally correlated with clozapine withdrawal. Nonetheless, there was a diagnostic conundrum. Mr. J presented with features of delirium (inattention, disorientation, acute onset visual hallucinations, and fluctuating course) and catatonia (negativism, periods of immobility, rigidity, and increased motor activity), as described in the Diagnostic and Statistical Manual of Mental Disorders, 5th edition [14]. However, these syndromes are not necessarily mutually exclusive. Given that both hyperactive and hypoactive delirium share motor features that overlap with catatonia, a catatonic subtype of delirium has been described in the literature [15], alternatively conceptualized as cooccurring delirium and catatonia [16-18], with descriptions of cases treated with memantine and ECT [17, 19]. The presence of suspected NMS in our patient further complicated his diagnosis, given that NMS often presents with features of catatonia and has previously been considered 
TABLE 1: Published case reports of syndromes precipitated by clozapine withdrawal treated with electroconvulsive therapy.

\begin{tabular}{|c|c|c|c|c|c|c|c|}
\hline Authors & $\begin{array}{l}\text { Psychiatric } \\
\text { diagnosis }\end{array}$ & Age & Sex & $\begin{array}{c}\text { Clozapine } \\
\text { daily dose } \\
(\mathrm{mg})\end{array}$ & $\begin{array}{l}\text { Reason for clozapine } \\
\text { discontinuation }\end{array}$ & Rebound syndrome & $\begin{array}{l}\text { Response to } \\
\text { ECT }\end{array}$ \\
\hline Bastiampillai et al. [2] & $\begin{array}{l}\text { Bipolar } \\
\text { Disorder }\end{array}$ & 51 & $\mathrm{~F}$ & $15-500$ & Nonadherence & Recurrent mania & Yes \\
\hline Cerit et al. [8] & Schizophrenia & 46 & M & 200 & Nonadherence & Catatonia & No \\
\hline Koch et al. [10] & Schizophrenia & 33 & $\mathrm{~F}$ & 250 & Leukopaenia & Catatonia & Partial \\
\hline Kumar et al. [11] & Schizophrenia & 29 & M & 250 & Febrile neutropaenia & Catatonia & Yes \\
\hline Mendhekar et al. [12] & Schizophrenia & 26 & M & 300 & Nonadherence & NMS, psychosis & Yes \\
\hline Bastiampillai et al. [13] & $\begin{array}{c}\text { Schizoaffective } \\
\text { disorder }\end{array}$ & 58 & $\mathrm{~F}$ & 150 & Nonadherence & $\begin{array}{l}\text { Catatonic, cholinergic, \& } \\
\text { serotonergic symptoms }\end{array}$ & Yes \\
\hline
\end{tabular}

as a subtype of malignant catatonia [20]. Though clozapinewithdrawal delirium has been previously described [3], and delirium of other causes treated with ECT [21], the present case appears to be the first reported clozapine-withdrawal delirium to have been successfully treated with ECT.

\section{Conflicts of Interest}

The authors declare no conflicts of interest.

\section{References}

[1] R. Durst, A. Teitelbaum, G. Katz, and H. Y. Knobler, "Withdrawal from clozapine: the rebound phenomenon," The Israel Journal of Psychiatry and Related Sciences, vol. 36, no. 2, p. 122, 1999.

[2] T. Bastiampillai, V. Juneja, and M. J. Nance, "Clozapine rebound mania," Australian \& New Zealand Journal of Psychiatry, vol. 48, no. 1, pp. 98-99, 2014.

[3] J. K. Stanilla, J. de Leon, and G. M. Simpson, "Clozapine withdrawal resulting in delirium with psychosis: a report of three cases," Journal of Clinical Psychiatry, vol. 58, no. 6, pp. 252-255, 1997.

[4] J. Bilbily, B. McCollum, and J. de Leon, "Catatonia Secondary to Sudden Clozapine Withdrawal: A Case with Three Repeated Episodes and a Literature Review," Case Reports in Psychiatry, vol. 2017, pp. 1-11, 2017.

[5] E. Stevenson, F. Schembri, D. M. Green, and J. D. Burns, "Serotonin syndrome associated with clozapine withdrawal," JAMA Neurology, vol. 70, no. 8, pp. 1054-1055, 2013.

[6] B. Margetic and B. Aukst-Margetic, "Neuroleptic malignant syndrome and clozapine withdrawal at the same time?" Progress in Neuro-Psychopharmacology \& Biological Psychiatry, vol. 29, no. 1, pp. 145-147, 2005.

[7] S. Ahmed, K. N. R. Chengappa, V. R. Naidu, R. W. Baker, H. Parepally, and N. R. Schooler, "Clozapine withdrawal-emergent dystonias and dyskinesias: a case series," Journal of Clinical Psychiatry, vol. 59, no. 9, pp. 472-477, 1998.

[8] C. Cerit, B. Tuzun, E. Akpinar, and E. Sahan, "Clozapine withdrawal catatonia refractory to ECT: a case report," Bulletin of Clinical Psychopharmacology, vol. 22, no. 3, pp. 275-277, 2012.

[9] M. K. Shields, T. Bastiampillai, and T. Mohan, "Managing clozapine discontinuation - Acute and chronic maintenance strategies," Australian \& New Zealand Journal of Psychiatry, vol. 46, no. 11, pp. 1104-1105, 2012.
[10] A. Koch, K. Reich, J. Wielopolski et al., "Catatonic Dilemma in a 33-Year-Old Woman: A Discussion," Case Reports in Psychiatry, vol. 2013, pp. 1-3, 2013.

[11] S. Kumar, S. Sur, and A. Singh, "Catatonia following abrupt stoppage of clozapine," Australian \& New Zealand Journal of Psychiatry, vol. 45, no. 6, article 499, 2011.

[12] D. N. Mendhekar, R. C. Jiloha, and H. S. Duggal, "Neuroleptic malignant syndrome precipitated by haloperidol following clozapine discontinuation," Australian \& New Zealand Journal of Psychiatry, vol. 39, no. 10, pp. 947-948, 2005.

[13] T. Bastiampillai, F. Forooziya, and R. Dhillon, "Clozapinewithdrawal catatonia," Australian \& New Zealand Journal of Psychiatry, vol. 43, no. 3, pp. 283-284, 2009.

[14] American Psychiatric Association, Diagnostic and Statistical Manual of Mental Disorders, DSM-5, American Psychiatric Association, Arlington, Va, USA, 5th edition, 2013.

[15] A. Francis and A. Lopez-Canino, "Delirium with catatonic features a new subtype?” Psychiatric Times, vol. 36, no. 2, pp. 239242, 2009.

[16] S. Grover, A. Ghosh, and D. Ghormode, "Do patients of delirium have catatonic features? An exploratory study," Psychiatry and Clinical Neurosciences, vol. 68, no. 8, pp. 644-651, 2014.

[17] K. Roy, S. J. Warnick, and R. Balon, "Catatonia Delirium: 3 Cases Treated With Memantine," Psychosomatics, vol. 57, no. 6, pp. 645-650, 2016.

[18] M. A. Oldham and H. B. Lee, "Catatonia vis-à-vis delirium: the significance of recognizing catatonia in altered mental status," General Hospital Psychiatry, vol. 37, no. 6, pp. 554-559, 2015.

[19] A. L. Santos Nunes and E. Cheniaux, "Delirium and Mania with catatonic features in a Brazilian patient: Response to ECT," The Journal of Neuropsychiatry and Clinical Neurosciences, vol. 26, no. 1, p. -E3, 2014.

[20] A. Roberto, S. Coelho, P. Sales, and I. Costa, "Malignant catatonia and neuroleptic malignant syndrome: two sides of the same coin?" European Psychiatry, vol. 29, p. 1, 2014.

[21] R. M. Nielsen, K. S. Olsen, A. O. Lauritsen, and H. C. Boesen, "Electroconvulsive therapy as a treatment for protracted refractory delirium in the intensive care unit-Five cases and a review," Journal of Critical Care, vol. 29, no. 5, pp. 881-881.e6, 2014. 


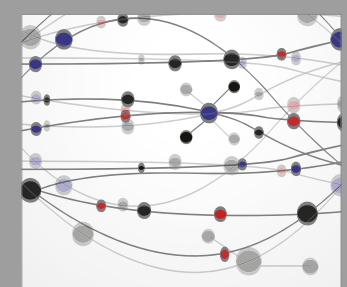

The Scientific World Journal
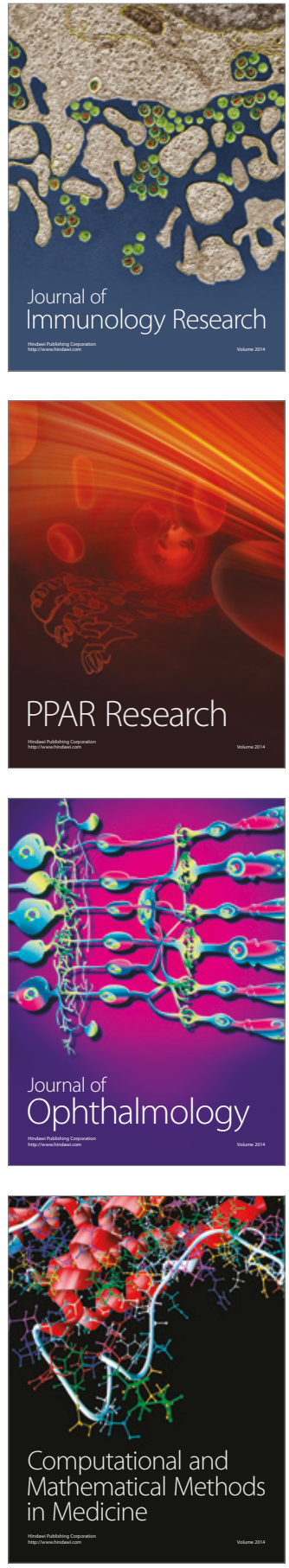

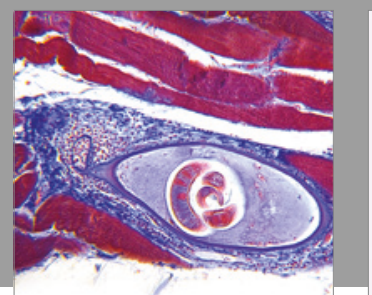

Gastroenterology Research and Practice
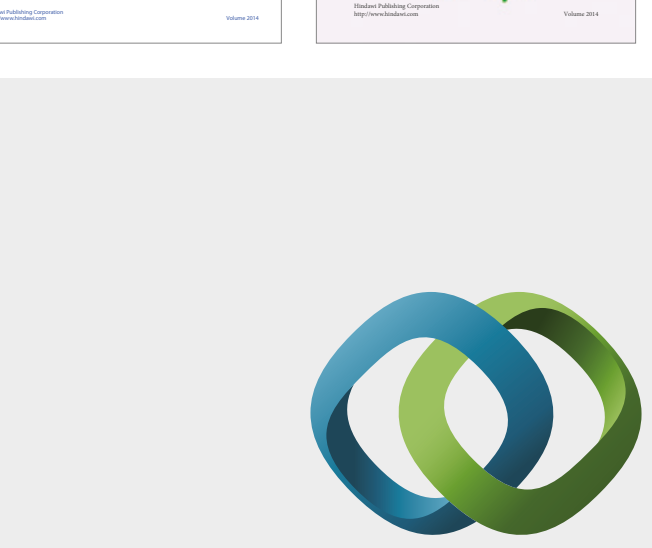

\section{Hindawi}

Submit your manuscripts at

https://www.hindawi.com
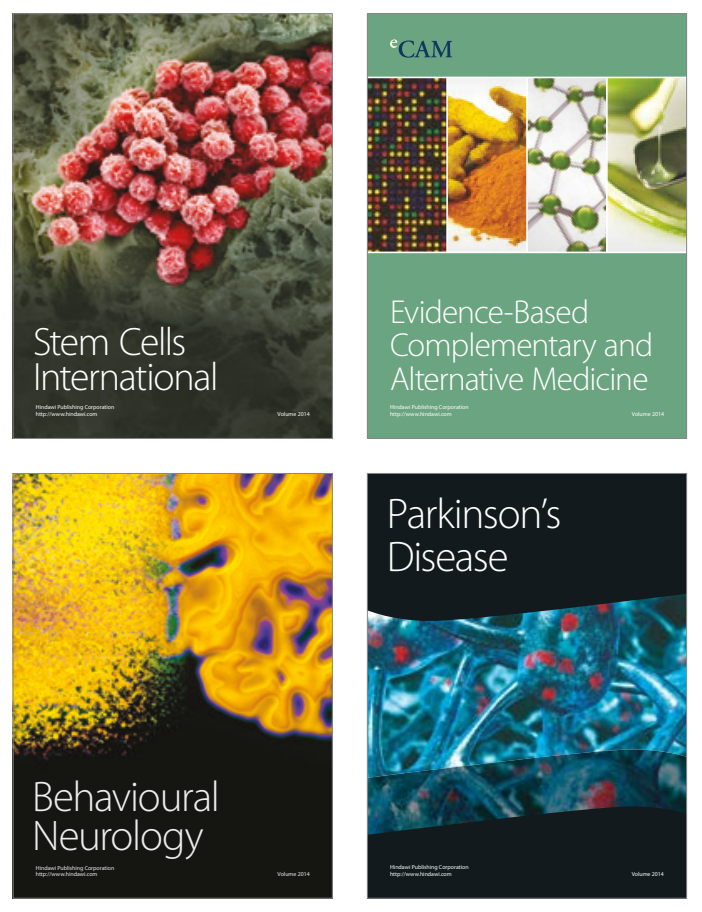
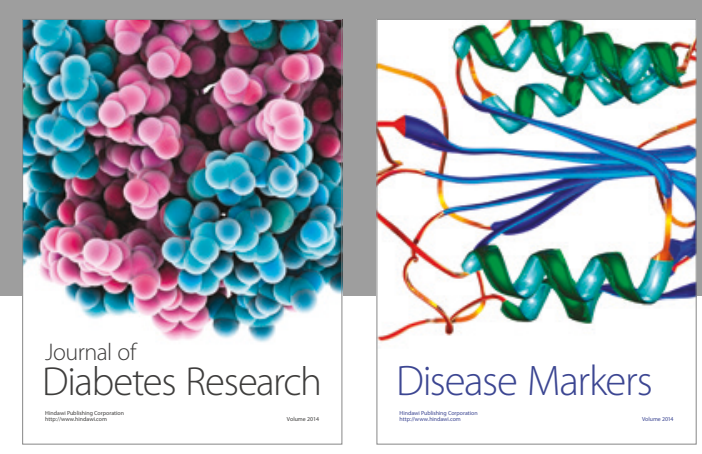

Disease Markers
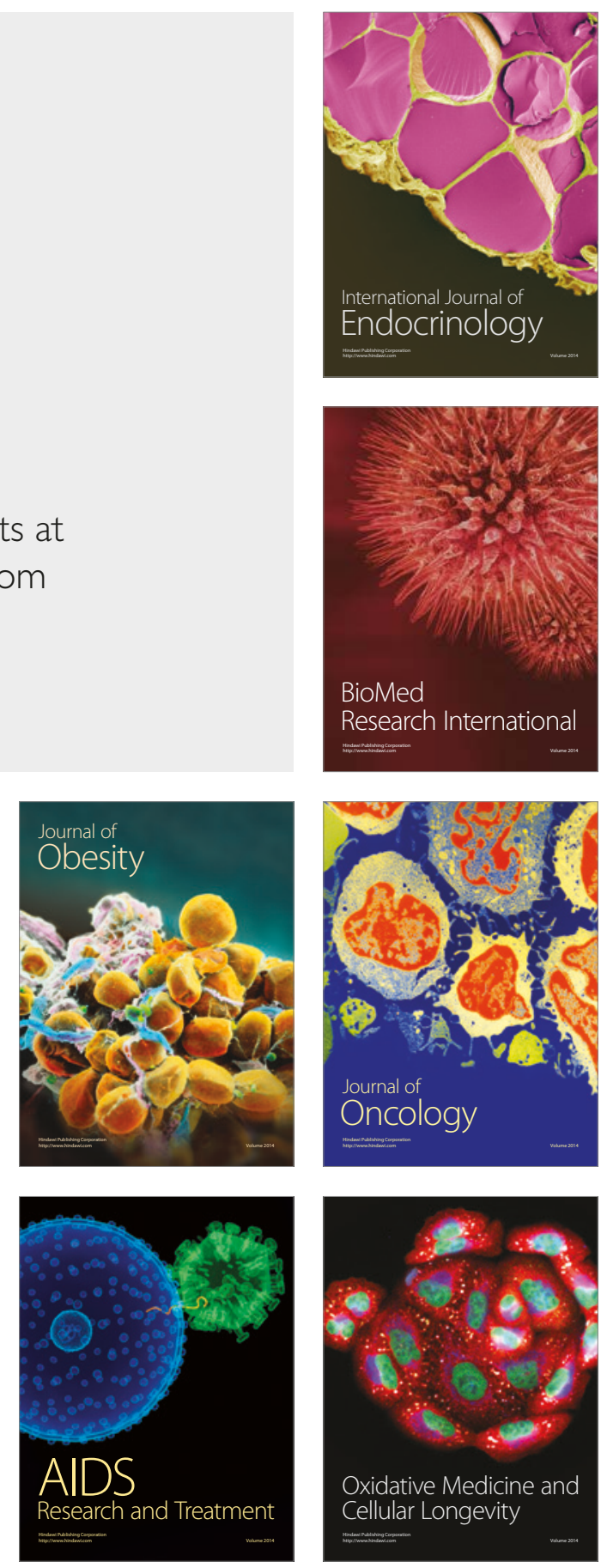